\title{
Analysis of quantitative PCR for the diagnosis of deletion and duplication carriers in the dystrophin gene
}

\author{
Stephen Abbs, Martin Bobrow
}

\begin{abstract}
A direct, non-radioactive method of quantitative PCR amplification has been investigated for the diagnosis of deletion and duplication carriers in the dystrophin gene. The simultaneous amplification of two loci, or several loci using multiplex PCR, allows for the direct comparison of relative amounts of products from normal homozygous loci and potentially heterozygous deleted/duplicated loci. Sufficient cycles of PCR are performed to enable visual analysis or densitometric quantification of products on ethidium bromide stained gels. The method has been verified in blind trials performed on known genotypes and by showing that under the conditions used the assay remains within the exponential phase of amplification.
\end{abstract}

The genetic defect responsible for the Duchenne and Becker muscular dystrophy phenotypes can be identified as a partial deletion of the dystrophin gene in approximately 50 to $60 \%$ of cases, ${ }^{1-3}$ or as a duplication in a further $6 \% .^{4}$ Detection of a deletion or duplication in the proband enables direct analysis of the mutated region in family members, greatly enhancing the accuracy of prenatal diagnosis and carrier risk determination. ${ }^{5}$

Several techniques have been described which display qualitative abnormalities in deletion and duplication carriers. Aberrant sized junction fragments can be detected by standard Southern analysis with cDNA probes, but only in approximately $10 \%$ of deletions. The frequency with which junction fragments are seen can be increased to virtually $100 \%$ by using a technique such as pulsed field gel electrophoresis, ${ }^{6}$ whole cosmid hybridisation, ${ }^{7}$ or amplification of reverse transcribed mRNA from whole blood. ${ }^{8}$ Additionally, in situ hybridisation has been used as a carrier test by showing failure of hybridisation of cosmids to deleted chromosomes. ${ }^{9}$

These qualitative analyses are obviously preferable to quantitative tests since the presence or absence of an aberrant fragment or hybridisation signal gives a definitive diagnosis of carrier status, with the exception of cases exhibiting germinal mosaicism. ${ }^{1011}$ However, the techniques used are technically demanding and time consuming. Fresh samples are required for most methods, and they cannot therefore be applied to banked DNA. The method of whole cosmid hybridisation is performed on standard DNA samples, but this test is restricted to regions of the $2.3 \mathrm{Mb}$ gene for which a series of fully characterised, overlapping cosmids are available, and is therefore at present only applicable to a limited number of deletions.

The standard method of diagnosing deletions has been Southern analysis with a series of cDNA probes each of which hybridises to between seven and 12 restriction fragments in a normal genotype. Quantitative analysis of Southern hybridisation, ${ }^{12}$ based on dosage differences observed between control loci and deleted loci, is the most widely used method for carrier detection, but this is also a technically demanding procedure for routine use. Meticulous care is required at all stages of the procedure in order to obtain blots of sufficiently good quality for reliable dosage analysis. Each sample should be analysed at least in duplicate with different restriction enzymes, along with adequate controls, and Prior et al ${ }^{13}$ have estimated that approximately $20 \%$ of analyses need to be repeated in order to achieve blots of the required quality. Although experienced analysts will often be able to distinguish between the 2:1 dosage ratio (control locus:deleted locus) observed in carriers and the $1: 1$ ratio in normals by visual inspection of an autoradiograph, densitometric quantification may be essential for detecting the $2: 3$ ratio expected in female duplication carriers. Where several hybridising fragments are analysed, as in the case of cDNA probes, observing a reduction in the intensity of bands that correspond with the deleted bands seen in the affected member of a family provides convincing evidence of the carrier status for a female. However, an apparent 1:1 ratio cannot be taken as an absolute indication of non-carrier status, since one of the many experimental variables may have prevented the autoradiographic signal giving a truly quantitative representation of genotype. Experimental data on the magnitude of these error rates, as observed with known genotypes, are not available.

There is increasing use of multiplex PCR, amplifying several exons simultaneously in a reaction, for deletion diagnosis. Previous reports on quantitative PCR have suggested that to ensure a standard reaction remains in its exponential (and hence quantitative) phase a limited number of cycles must be used which will not produce sufficient product to visualise on ethidium bromide stained gels. A PCR technique has been reported by Gilliland $e t a l^{14}$ for quantifying the genomic copy number of 
the granulocyte-macrophage colony stimulating factor (GM-CSF) gene. This involved the competitive amplification of a CDNA sequence with its genomic DNA sequence that differed by possessing a small intron. Since the two templates were essentially the same, the relative amplifications of the two sequences remained constant for sufficient PCR cycles to visualise the products with ethidium bromide. This technique requires an initial accurate spectrophotometric determination of DNA concentrations. It would not be applicable in the dystrophin gene where introns are large and constitute $99 \%$ of the total sequence.

A PCR technique has been applied to carrier determination in $\mathrm{DMD} / \mathrm{BMD}^{13}$ which uses an alternative method of detecting products from a limited number of amplification cycles, thereby ensuring the reaction has remained in its exponential phase. This involved the simultaneous amplification of potentially deleted and control loci, electrophoresis and Southern blotting of PCR products, followed by hybridisation with radioactively labelled allele specific oligonucleotides, and densitometric analysis of the resulting autoradiographs. An alternative method involves the use of fluorescently labelled primers in a reaction, which can be detected and quantified electronically, using, for example, an automatic sequencing machine. ${ }^{15}$

These methods either complicate the basic PCR technique or require expensive equipment that will not be available to the majority of diagnostic laboratories. We have investigated a simpler, more direct, non-radioactive method of quantitative PCR amplification from genomic DNA, and determined the accuracy of the procedure before its application in the diagnosis of potential female carriers of DMD/BMD.

\section{Methods}

\section{PCR AMPLIFICATION}

Concentrations of genomic DNA were measured by fluorometric assay, and samples were diluted to a concentration of $25 \mathrm{ng} / \mu \mathrm{l}$. Then $125 \mathrm{ng}$ of diluted DNA was added to each reaction tube containing $0.5 \mu \mathrm{mol} / 1$ of each oligonucleotide primer (for the simultaneous amplification of either two, seven, or 10 exon specific loci in the dystrophin gene); $1 \times$ Taq DNA polymerase buffer $(67 \mathrm{mmol} / 1$ Tris- $\mathrm{HCl}, \mathrm{pH} 8.8,16.6 \mathrm{mmol} / 1 \quad\left(\mathrm{NH}_{4}\right)_{2} \mathrm{SO}_{4}$, $6.7 \mathrm{mmol} / 1 \mathrm{MgCl}_{2}, 170 \mu \mathrm{g} / \mathrm{ml} \mathrm{BSA}, 10 \mathrm{mmol} / 1$ 2-mercaptoethanol); $0.5 \mathrm{mmol} / 1 \mathrm{dNTPs}$ and Taq DNA polymerase (Amplitaq, Cetus) $(1 \cdot 2$ units for two loci, three units for seven and 10 loci) in a total reaction volume of $30 \mu 1$ (two loci) or $50 \mu \mathrm{l}$ (seven and 10 loci). PCR amplification was performed on a Techne II Thermal Cycling Block, under the following conditions: $94^{\circ} \mathrm{C}$ for five minutes, followed by 23 cycles of $94^{\circ} \mathrm{C}$ for 48 seconds, $60^{\circ} \mathrm{C}$ for 48 seconds, $72^{\circ} \mathrm{C}$ for three minutes, with a final extension of $72^{\circ} \mathrm{C}$ for five minutes.
INCORPORATION OF LABEL IN PCR

In order to plot the progress of PCR amplification, a DNA sample from a normal female was amplified with a $5^{\prime}$ multiplex reaction (see test 3 below) for varying numbers up to 33 cycles of PCR. The amplifications were performed under the precise conditions as used for the assays described below, with the inclusion of $2 \mu \mathrm{l}$ of $\left[\alpha-{ }^{32} \mathrm{P}\right] \mathrm{dCTP}(10 \mathrm{mCi} / \mathrm{ml}, 3000 \mathrm{Ci} /$ $\mathrm{mmol}$ ) radioactive label in each reaction. The proportion of label incorporated into the PCR products was measured by the method of differential trichloroacetic acid precipitation ${ }^{16}$ and quantification in a scintillation counter. Results were plotted against cycle number.

\section{ASSAYS FOR CARRIER STATUS}

Blind trials were performed on genomic DNA samples of known genotype in order to ascertain error rates for the methods. Samples were taken from females known to carry a deletion within the dystrophin gene (as a consequence of having a family history of the disease and themselves having at least one affected son with a deletion) and from presumptively normal homozygous females (colleagues, and relatives of cystic fibrosis patients). Samples were coded by a colleague before three independent experiments.

A fourth experiment was performed in order to assess the ability of the PCR technique to detect carriers of duplications. Samples were analysed from nine members of a large family (P.18317) in which the affected boy had a duplication of exon 17. The samples consisted of four non-carriers, five carriers, and one affected male. The carrier status of females in this family had previously been ascertained by quantitative Southern cDNA hybridisation, linkage analysis with polymorphic markers that closely flank the mutation, CK measurements (unpublished data), and amplification of reverse transcribed mRNA, ${ }^{8}$ all of which gave concordant diagnoses.

\section{Test 1}

Nine samples from normal females and 11 samples from DMD carriers known to be heterozygous for deletions within the central deletion 'hotspot' were subjected to the simultaneous amplification of two loci, a test locus (exon 47) for which the DMD carriers were known to be deleted, and a control locus (exon 13 or 9) which was assumed to be present in the normal two copies in all samples. Each sample was amplified in triplicate, initially with primers for exon 47 and exon 13 (series 1a), and then again (after recoding) in triplicate with a different pair of primers for the amplification of exon 47, along with exon 9 (series 1b). Primer sequences were designed using the published cDNA sequence ${ }^{17}$ or taken from Beggs et $a l,{ }^{18}$ and are available from the authors. 


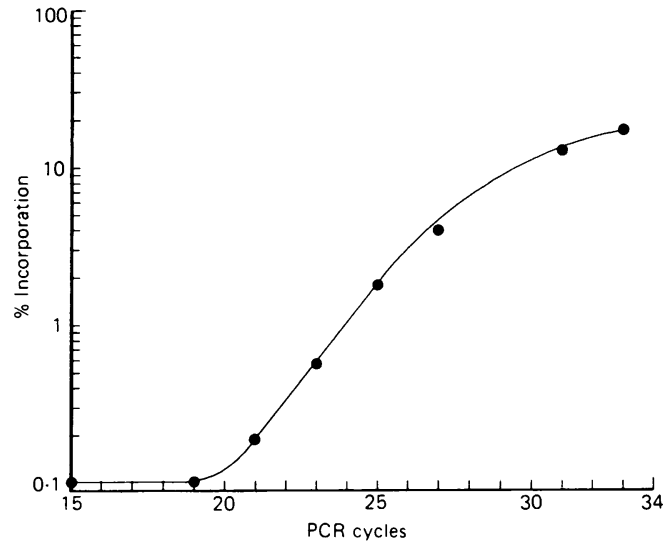

Figure 1 Graph showing the percentage of radioactive label (log scale) incorporated into a multiplex amplification, plotted against number of PCR cycles.

Test 2

Ten samples from normal females and 10 samples heterozygous for deletions in the central 'hotspot' region were subjected to multiplex PCR amplification ${ }^{19}$ of exons $43,44,45$, $47,48,50,51,52,53$, and $60\left(3^{\prime}\right.$ multiplex reaction described in Abbs et al, ${ }^{20}$ with the omission of exon 42). Amplifications were performed in duplicate.

Test 3

Test 2 was essentially repeated using a multiplex reaction that simultaneously amplifies exons $1,3,4,6,8,13$, and $19\left(5^{\prime}\right.$ multiplex reaction $^{20}$ ). A different set of 10 normal samples and 10 samples heterozygous for deletions at the $5^{\prime}$ end of the dystrophin gene were analysed.

\section{Test 4}

Samples being tested for the presence of a duplication of exon 17 were analysed in the same blind manner as described in test 1 , using primers for exon 17 and exon 8 as a control (primers from Chamberlain et $a l^{1921}$ ).

\section{ANALYSIS OF PCR PRODUCTS}

Tests 1 and 4

A sample of $12 \mu \mathrm{l}$ of products was removed from each reaction and electrophoresed on 3 or $4 \%$ NuSieve agarose gels, stained with ethidium bromide, and photographed under UV light onto Polaroid 665 type film producing $950 \times 730 \mathrm{~mm}$ negatives. Each lane on a negative was subjected to densitometric analysis and integration (using a BioRad VD620 Video

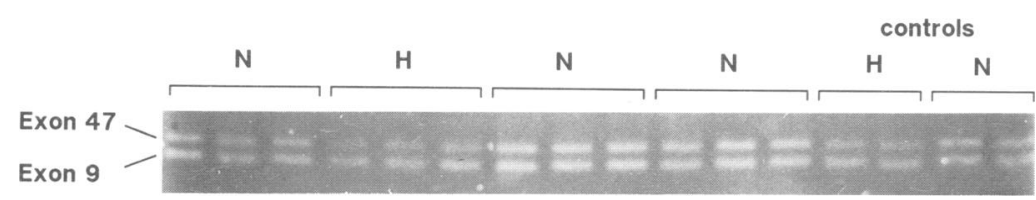

Figure 2 Products of amplification from exon 9 (control locus) and exon 47 (test locus) on an ethidium bromide stained agarose minigel. Samples are shown in triplicate; a control exon 47 deletion heterozygote $(H)$ and normal (N) sample are shown on the right, along with three unknown samples and their resulting diagnoses.
Densitometer) to quantify the product bands resulting from PCR amplification. A ratio of the area under the peak produced by the normal control locus to that produced by the test (potentially deleted (test 1) or duplicated (test 4)) locus was calculated for each reaction. Mean values obtained from triplicate analyses on each sample were plotted graphically and a diagnosis of deletion heterozygote, duplication carrier, normal, or intermediate/unknown was assigned to the samples, based on the distribution of the plotted results. Samples belonging to deletion heterozygotes were expected to produce a peak ratio value (control locus:test locus) of twice the value observed in normal samples, female carriers of the duplication a ratio of approximately 2:3 (control locus:duplicated locus), and the affected male with a duplication 1:2.

\section{Tests 2 and 3}

A sample of $12 \mu \mathrm{l}$ of product from each multiplex reaction was electrophoresed on a $5 \%$ (test 2 ) or $7 \%$ (test 3) horizontal acrylamide gel, stained, and photographed as above. The samples were typed from the photographs by three experienced investigators.

\section{Results}

INCORPORATION OF LABEL

Results showing the incorporation of radioactive label at different numbers of PCR cycles are plotted in fig 1 . The amplification is shown to be within the limits of the exponential phase at the 23 cycle stage that has been used in the assays for carrier status.

\section{Test 1}

An example of products from the amplification of exons 9 and 47 is shown in fig 2 . Results from the densitometric quantification are shown graphically in fig 3. The classification of samples was based on peak area ratios falling into two groups in each set of reactions. The mean peak area ratio (control:test locus) of samples classified as carriers was approximately twice that of those classified as noncarriers.

Sixteen of 20 samples resulted in consistent classification between series $1 \mathrm{a}$ and $1 \mathrm{~b}$ and were classified as deletion heterozygotes or normals. Of the total 40 tests in series 1a plus $1 b$ (on 20 samples), 36 gave the correct diagnosis, two were unassignable, and two were incorrect, giving an overall error rate of $2 / 38$ $(5 \cdot 3 \%)$. Four samples gave either inconsistent or intermediate results between $1 \mathrm{a}$ and $1 \mathrm{~b}$. Repeating the amplifications on these four samples produced consistent results for one sample, but inconsistent or intermediate results for the remaining three. This was clarified by performing a further analysis with exon 45 (also known to be deleted in the group of carriers) and exon 3 as a control, and determining the status of these four samples by taking the majority result (of either carrier or noncarrier) from the three series of tests (table). By 
Assays for deletion status
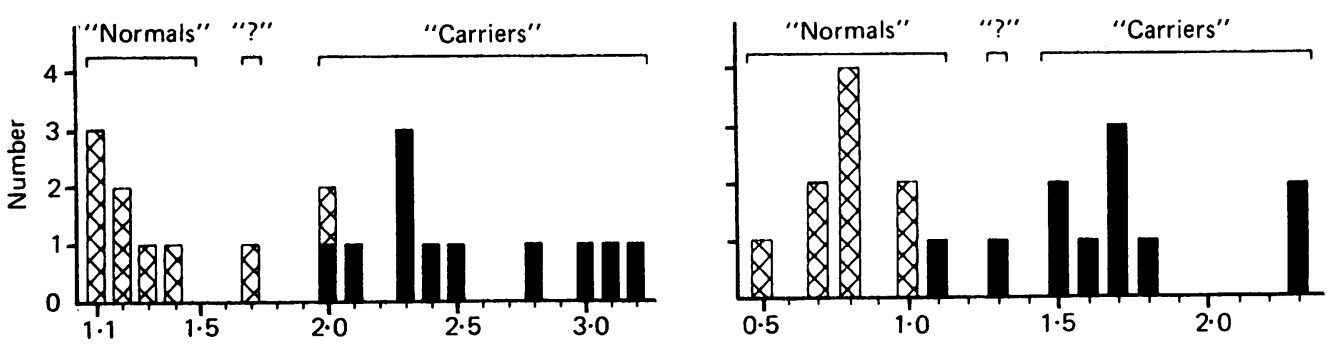

Assay for duplication status Exon 8 / Exon 17

Test 4
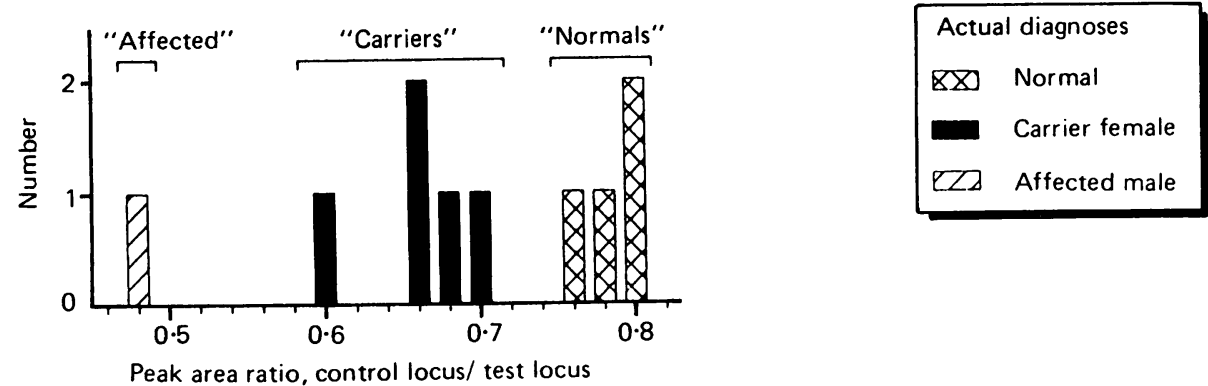

Figure 3 Graphical representation of results obtained from amplifications in tests $1 a, 1 b$, and 4. Actual genotypes are represented by the different bar shadings. Diagnoses of carrier, normal, intermediate (?), or affected, assigned to sample groups based on their peak area ratios, are shown by the brackets above the bars.

using this third test (1c), all 20 samples were correctly diagnosed as deletion carriers or normals.

\section{Test 2}

Products of amplification from the $3^{\prime}$ multiplex reaction are shown in fig 4 (top). Samples were typed as being normal if all bands were of similar intensity, or carrying a deletion if one or more bands were of markedly reduced intensity. The carrier status of all 20 samples was correctly identified by all three investigators who independently typed the photograohs by visual comparison of the relative intensities of product bands within each lane. The extent of deletions in the 10 carriers was also determined correctly by all three investigators.

Test 3

Fig 4 (bottom) shows products of amplification from the $5^{\prime}$ multiplex reaction. All 20 samples were correctly identified as normals or

Results obtained on the four samples giving inconsistent results in the initial series of amplifications ( $1 a$ and $1 b$ ).

\begin{tabular}{llccc}
\hline Sample & 1a & 1b & 1c & Conclusion \\
\hline 4 & I I & N N & I & N \\
9 & H H & N I & H & H \\
10 & H H & N N & N & H \\
11 & H H & I H & H & H
\end{tabular}

Series $1 \mathrm{a}=$ amplification of exon 13 and 47 .

Series $1 \mathrm{~b}=$ amplification of exon 9 and 47 (different primers) Series $1 c=$ amplification of exon 3 and 45 .

Conclusion = status assigned, based on majority result.

$\mathrm{I}=$ Intermediate result. $\mathrm{N}=$ Normal result. $\mathrm{H}=$ Heterozygou result. deletion heterozygotes by all three investigators. The extent of nine out of 10 deletions was correctly determined by one investigator, and six were correctly determined by two investigators. All three investigators incorrectly typed the extent of deletion in one sample (sample 14, fig 4). This sample clearly appeared to carry a deletion of exons 8 and 13 . The original multiplex reaction performed on the affected male in the family showed a deletion of exon 8, but exon 13 had amplified weakly in comparison with other intact exons.

Test 4

The analysis of family members with the exon 17 duplication correctly distinguished noncarriers, female carriers (peak area ratio of 2:3, control:test locus), and an affected male (1:2 ratio). Results are shown in fig 3.

\section{Discussion}

We have investigated two quantitative PCR techniques that directly compare the ratio of products resulting from the simultaneous amplification of test loci and internal normal control loci in the diagnosis of DMD deletion heterozygotes.

In the first test (1) two loci were coamplified in each reaction. This produced an initial error rate of $5 \cdot 3 \%$. However, by performing a third amplification reaction (1c) for samples giving ambiguous results the final frequency of error should be considerably reduced, and using this procedure all 20 samples were correctly diagnosed as carrying a deletion or being normal. This suggests an overall error rate of less than $5 \%$. Further tests are required to estimate this figure more accurately. 


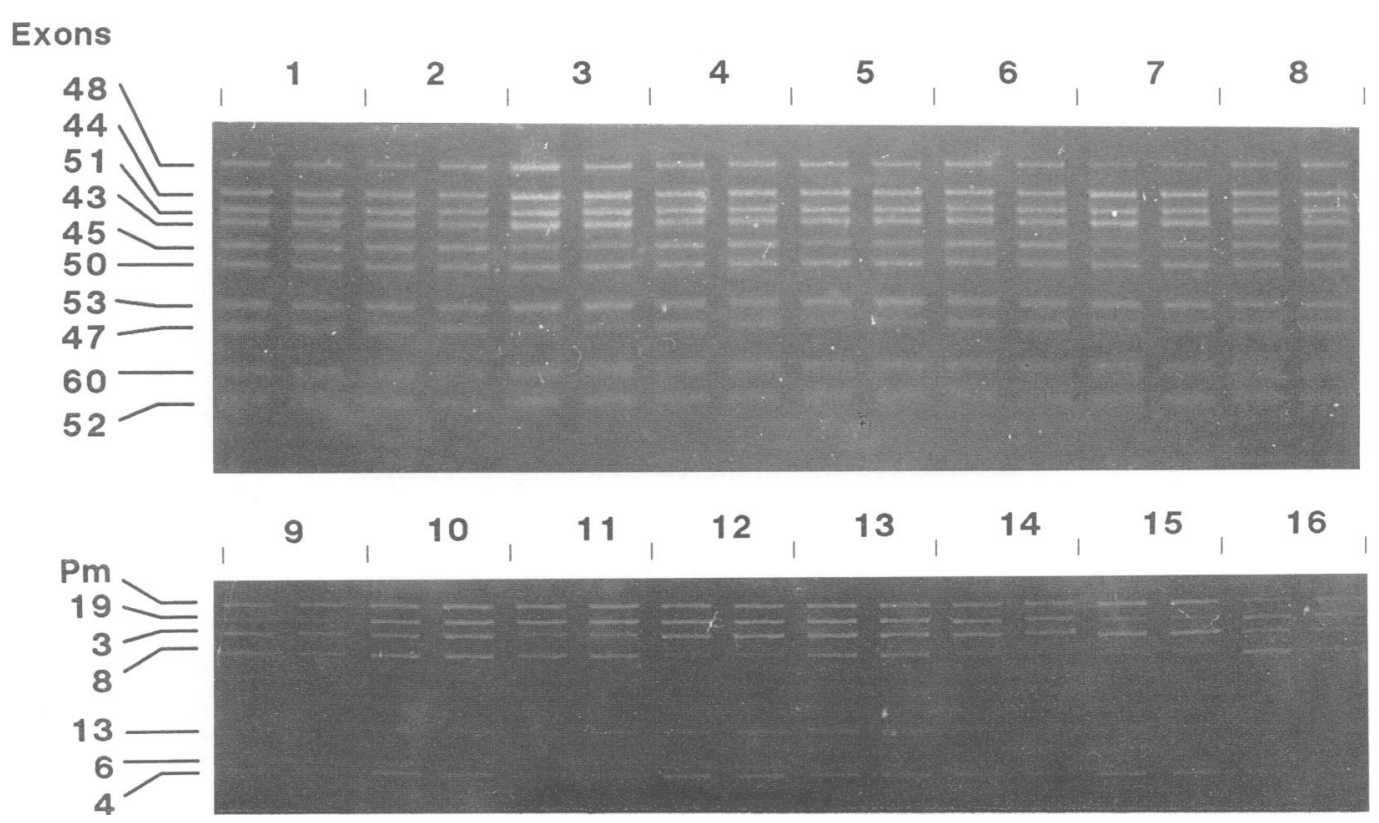

Figure 4 Products of amplification from the $3^{\prime}$ (top) and 5' (bottom) multiplex reactions. Exons amplified are shown on the left ( $\mathrm{Pm}=$ muscle specific promotor plus a quarter of exon 1). Samples (run in duplicate) are normal controls $(N)$ or deletion heterozygotes $(H)$ for the exons listed: (1) $N$, (2) $N,(3) H 45,47,(4) N$, (5) $H 45,47$, (6) $N$, (7) $H 45,47,48$, , (8) $N$, (9) $H 13,19$, (10) $N$, (11) $H 3,4,6,(12) H 8,(13) N,(14) H 8,(15) H 8$, $13,19,(16) N$.

The second and third tests used two PCR multiplex reactions that are routinely used for the detection of deletions in affected patients. ${ }^{20}$ The presence of several PCR products within a multiplex reaction facilitates comparison of relative product intensities making the visual interpretation of dosage relatively straightforward for experienced analysts. Deletions involving a single exon within a multiplex reaction are less obvious than deletions of more than one exon (although two cases of single exon deletion carriers were correctly identified in the tests).

The carrier status of all 40 samples (20 analysed with each multiplex reaction) was correctly determined by all three investigators. The typing was performed with no knowledge of the extent of the deletions being analysed. With the $3^{\prime}$ multiplex reaction (test 2 ) it was possible for all three investigators to type correctly which exons were deleted in each carrier. This was not the case with the $5^{\prime}$ reaction (test 3), although all investigators did correctly ascertain the carrier status for the 20 samples. The mistypings in the extent of deletions were thought to be the result of the greater separation of some products on a gel making comparative analysis of bands within a track more difficult, as compared with the more evenly spaced products from the 3 ' multiplex. However, the mistyping by all three investigators of the extent of deletion of one sample (sample 14 , fig 4) is thought to have resulted from an inherent failure in the PCR method rather than investigator mistyping. The weak amplification of the exon (in both the carrier mother and her affected son) may have resulted from a sequence variation within one of the priming sites reducing effective priming at this locus. ${ }^{2022}$
Mistyping the extent of a deletion in a carrier would not be a problem for the majority of diagnoses since a sample is available from an affected male in approximately $80 \%$ of families (unpublished data), and the size of deletion being tested for will be known. In the remaining $20 \%$, quantitative multiplex PCR can be used as an initial test, but should be supplemented by another method to confirm the results. Similar confirmation of multiplex dosage analysis should also be sought in the $28 \%$ of deletion cases that involve the absence of a single exon product, ${ }^{20}$ rather than relying on quantitative estimation of that single PCR product.

The ability of the PCR technique to detect duplications has also been shown. Since a 2:1 ratio is readily observed on multiplex PCR in female deletion carriers, it should also be possible to detect the 1:2 ratio expected in male duplication carriers. The ability to detect an extra $6 \%$ of mutations ${ }^{4}$ would be of benefit in the genetic counselling of these families. Detection of female duplication carriers would require quantitative densitometric analysis for reliable interpretation of amplification products.

Here we have shown that the PCR technique can be used quantitatively without the complex procedures that were previously thought to be necessary. Under the conditions described, at 23 cycles of PCR amplification the reaction was shown to be within the limits of the exponential phase, and dosage was readily visualised at that stage.

Two factors affect the accuracy of quantitation in a PCR amplification. Firstly, the relative amplifications of products to be compared should be proportional to the original template. In a multiplex reaction this depends 
on all primers being present in equal molarities and optimising the annealing temperature to ensure even amplification of all loci. The products of amplification should also be assayed at a stage where no component of the reaction is limiting (dNTPs, primers, and especially enzyme) nor inhibitory (for example, accumulation of product). ${ }^{23}$ Secondly, the accuracy is dependent on the sensitivity of the assay used. Accurate quantification of ethidium bromide staining is critically dependent on the amount of DNA loaded onto a gel. Overloading of products results in increased intensity of bands and markedly reduces the ability to distinguish differences in dosage. Thus to ensure a quantitative assay the amount of product loaded on a gel should be limited, and gels should be of optimum quality, with sharp, straight bands of equal thickness. Other assays such as those that use fluorescence for product detection may have a better range of sensitivity, but the technical equipment required will not be available for many laboratories.

The methods reported here can in principle be applied to any given locus for quantification of genomic copy number, provided sufficient sequence data are available. Provided rigorous controls are used, we conclude that this direct quantitative PCR technique can be a reliable method for diagnosing carriers of deletions and duplications in the dystrophin gene.

We thank Suzanne Clark for her invaluable assistance and Drs David Bentley and Christopher Mathew for helpful discussion. The study was supported by the Muscular Dystrophy Group of Great Britain and Northern Ireland, the Generation Trust, and the Spastics Society.

1 Koenig M, Hoffman EP, Bertelson CJ, Monaco AP, Feener , Kunkel LM. Complete cloning of the Duchenn muscular dystrophy (DMD) cDNA and preliminary genomic organisation of the DMD gene in normal and affected individuals. Cell 1987;50:509-17.

2 Forrest SM, Cross GS, Speer A, Gardner-Medwin D, Burn J, Davies KE. Preferential deletion of exons in Duchenne and Becker muscular dystrophies. Natur 1987;329:638-40.

3 Hodgson SV, Hart K, Abbs S, et al. Correlation of clinica and deletion data in Duchenne and Becker muscular dystrophy. F Med Genet 1989;26:682-93.

4 Hu X, Ray PN, Murphy E, Thompson MW, Worton RG. Duplicational mutation at the Duchenne muscular dystrophy locus: its frequency, distribution, origin and phenotype/genotype correlation. Am 7 Hum Genet 1990;46:682-95.

5 Hodgson SV, Bobrow M. Carrier detection and prenatal diagnosis in Duchenne and Becker muscular dystrophy. $\mathrm{Br}$ Med Bull 1989;45:719-44.

6 Den Dunnen JT, Bakker E, Klein Breteler EG, Pearson PL van Ommen GJB. Direct detection of more than $50 \%$ of the Duchenne muscular dystrophy mutations by field inversion gels. Nature 1987;329:640-2.

7 Blonden LAJ, den Dunnen JT, van Passen HMB, et al. High resolution deletion breakpoint mapping in the DMD gene by whole cosmid hybridization. Nucleic Acids Res 1989;17:5611-21.

8 Roberts RG, Bentley DR, Barby TM, Manners E, Bobrow M. Direct diagnosis of carriers of Duchenne and Becker muscular dystrophy by amplification of lymphocyte muscular dystrophy by amp

9 Ried T, Mahler V, Vogt P, et al. Direct carrier detection by in situ suppression hybridisation with cosmid clones of the Duchenne/Becker muscular dystrophy locus. Hum Genet 1990;85:581-6.

10 Bakker E, van Broeckhoven $\mathrm{Ch}$, Bonten EJ, et al. Germline mosaicism and Duchenne muscular dystrophy mutations. Nature 1987;329:554-6.

11 Darras BT, Francke U. A partial deletion of the muscular dystrophy gene transmitted twice by an unaffected male. Nature 1987;329:556-8.

12 Mao Y, Cremer M. Detection of Duchenne muscular dystrophy carriers by dosage analysis using the DMD cDNA clone 8. Hum Genet 1989;81:193-5.

13 Prior TW Papp AC Snyder PJ, et al. Determination of carrier status in Duchenne and Becker muscular dystrophies by quantitative polymerase chain reaction and allele-specific oligonucleotides. Clin Chem 1990; 36:2113-7.

14 Gilliland G, Perrin S, Blanchard K, Bunn F. Analysis of cytokine mRNA and DNA: detection and quantitation by competitive polymerase chain reaction. Proc Natl Acad Sci USA 1990;87:2725-9.

15 Chamberlain JS, Gibbs RA, Ranier JE, Caskey CT. An integrated approach to Duchenne muscular dystrophy diagnosis via multiplex polymerase chain reaction. $A m \mathcal{F}$ Hum Genet 1989;45:134A.

16 Sambrook J, Fritsch EF, Maniatis T. Molecular cloning: a laboratory manual. 2nd ed. New York: Cold Spring Harbor Laboratory Press, 1989.

17 Koenig M, Monaco AP, Kunkel LM. The complete sequence of dystrophin predicts a rod-shaped cytoskeletal protein. Cell 1988;53:219-28.

18 Beggs AH, Koenig M, Boyce FM, Kunkel LM. Detection of $98 \%$ of $\mathrm{DMD} / \mathrm{BMD}$ deletions by polymerase chain reaction. Hum Genet 1990;86:45-8.

19 Chamberlain JS, Gibbs RA, Ranier JE, Nguyen PN, Caskey CT. Deletion screening of the Duchenne muscular dystrophy locus via multiplex DNA amplification. Nucleic Acids Res 1988;16:11141-56.

20 Abbs S, Yau SC, Clark S, Mathew CG, Bobrow M. A convenient multiplex PCR system for the detection of dystrophin deletions: a comparative analysis with cDNA hybridisation shows mistypings by both methods. $\mathfrak{F}$ Med hybridisation shows mist
Genet 1991;28:304-11.

21 Chamberlain JS, Gibbs RA, Ranier JE, Caskey CT. Multiplex PCR for the diagnosis of Duchenne muscular dystrophy. In: Innis $M$, Gelfand D, Sninski J, White T, eds. PCR protocols: a guide to methods and applications. Orlando: Academic Press, 1990:272-81.

22 Fujimura FK, Northrup H, Beaudet A, O'Brien WE. Genotyping errors with the polymerase chain reaction. $N$ Engl f Med 1990;322:61.

23 Innis MA, Gelfand DH. Optimization of PCRs. In: Innis $\mathrm{M}$, Gelfand D, Sninski J, White T, eds. PCR protocols: $a$ guide to methods and applications. Orlando: Academic guide to methods
Press, 1990:3-12. 